\title{
Experimental Study On Digital Design Of Doppler Ultrasound With Coded Excitation
}

\author{
Shaoxing $\mathrm{Li}^{1,2,3, \mathrm{a}}$, Tianjiong Zhang ${ }^{1,2,3, \mathrm{~b}}$, Jie $\mathrm{Li}^{1,2,3, \mathrm{c}}$, Ming Dai ${ }^{1,2,3, \mathrm{~d}}$, \\ Xin Chen ${ }^{1,2,3, e}$, and Zhengdi Qin ${ }^{1,2,3, f}$ \\ ${ }^{1}$ National-Regional Key Technology Engineering Laboratory for Medical Ultrasound, Shenzhen \\ 518060 , Guangdong, China; \\ ${ }^{2}$ Guangdong Key Laboratory for Biomedical Measurements and Ultrasound Imaging, Shenzhen \\ 518060 , Guangdong, China; \\ ${ }^{3}$ Department of Biomedical Engineering, Health Science Center, Shenzhen University, Shenzhen \\ 518060 , Guangdong, China. \\ aIsxszu2014@163.com, b873209976@qq.com,'1989lijie.1989@gmail.com, \\ daily2020@sina.com, ${ }^{\mathrm{e}}$ chenxin@szu.edu.cn, ${ }^{\mathrm{f}}$ finyan@szu.edu.cn
}

Keywords: Digital design; Doppler ultrasound; Blood flow; Coded excitation

\begin{abstract}
Full digital design with coded excitation for Doppler ultrasound system is proposed to offer full-depth examination, and to improve system SNR and penetration. The experimental platform is developed based on a Doppler phantom and a transcranial Doppler system. Raw radiofrequency signals are collected from the Doppler phantom using the transcranial Doppler system, which alternately transmits non-coded and coded pulses. Software-based digital signal processing techniques are well used to reduce the scale of hardware. The results tell us that by using this design, full-depth examination and some 20dB of SNR's improvement are achieved. Penetration ability and sensitivity become much stronger compared with traditional systems. This work will facilitate blood flow examination in clinical diagnosis.
\end{abstract}

\section{Introduction}

Doppler ultrasound is a noninvasive procedure used to measure blood flow by bouncing ultrasound waves off of red blood cells. This test can determine how blood flows through a blood vessel by measuring the rate of change of its frequency. It helps doctors evaluate blood flow through major arteries and veins, such as those of the arms, legs, neck and brain. During pregnancy, Doppler ultrasound may be used to look at blood flow of the fetus as well. During Doppler ultrasound, gel will be applied to the area to allow ultrasound waves to pass through, before a transducer is placed on the area and move along the skin to produce images. The transducer sends and receives ultrasound waves that are amplified through a microphone. The ultrasound waves bounce off solid objects, including blood cells. The movement of blood cells causes a change in pitch of the reflected ultrasound waves (called Doppler Effect). If there is no blood flow, the pitch does not change. Information from the reflected ultrasound waves can be processed by a block of circuit and computer to provide graphs or pictures that represent the flow of blood through the blood vessels. Especially in transcranial Doppler (TCD) diagnosis, due to the inadequate depth information provided by the conventional Doppler ultrasound, it is not easy for an inexperience doctor to find the vessels in the brain.

Due to the frequency-dependent attenuation with ultrasound waves propagating in tissue, reflected ultrasound waves from tissue and vessels far from transducer are weak. The SNR of the reflected ultrasound waves from long distance vessels and tissues carrying Doppler information is inadequate so that the measurement of the blood flow becomes difficult. To improve the penetration, increasing peak acoustic power is a direct method. However, it is restricted because of instantaneous pressure effects, such as cavitation and mechanical index (MI). Because the average power delivered by most ultrasound systems compared to maximum limited levels often is very small. Spread spectrum technique (coded excitation) is a feasible method which can greatly increase the average power without affecting the peak power. It can improve the penetration and SNR of the 
Doppler ultrasound. Such improvements allow increased operating frequencies, and hence enhanced axial resolution [1].

The coded excitation has been well used in radar system to improve SNR at modest peak power levels compared to conventional pulse techniques. Its applications into clinical ultrasound also have been studied for about 20 years. It developed slowly for practical implementation issues such as the nonlinear ultrasound propagation in the human body. O'Donnell [1] revealed that SNR improvement of 15-20 dB can be achieved by coded excitation before reaching particular intensity limits (1992). In addition, Richard Y. Chiao and Xiaohui Hao [2] quantified tradeoffs between penetration and resolution, compared chirp and Golay code performance and illustrated the applications of coded excitation in B-mode, harmonic and flow imaging (2005). Joanne Cowe et al. $[3,4]$ proposed a method of using chirp to improve axial resolution of TCD systems and track the motion of emboli. Xiang Lei et al. [5] suggested that the system using Barker-coded excitation obtained a $2.78 \mathrm{~dB}$ improvement in measured embolus-to-blood (2009). Zhengdi Qin introduced a novel coding scheme named Truncated Long Code (TLC), and successfully used in Doppler Ultrasound system $[6,7,8]$.

In this letter, a full digital design of signal processing techniques for blood flow examination using Barker-coded excitation is introduced. Compared with conventional Doppler ultrasound systems, firstly, full digital design provides adequate Doppler information so as to display full-depth blood flows of vessels in ultrasound waves' propagation direction, and secondly coded excitation can be easily used in full digital design to obtain the increase in SNR of more than 20dB. This will improve the penetration ability and system sensitivity, and make blood flow measurement much easier even for an inexperienced person. Especially in the examination of transcranial blood flows, advantages are performed more obviously due to the strong attenuation when ultrasound propagates through the skull. To study transcranial propagation of ultrasound waves, a bone sample is placed between the transducer and the Doppler phantom to simulate the circumstances of transcranial.

\section{Methodology}

Experiment Set-up. The in-vitro work described in this study is developed based on a TCD ultrasound system board. Signal transmission circuits, RF signal receive circuits, $16 \mathrm{MHz}$ system clock source, transmit gate and pre-amplifier on the board are used in this experimental system. Figure 1 schematically illustrates the experimental system. A $2 \mathrm{MHz}$ weak focused single-element transducer ( $10 \mathrm{~cm}$ focal depth, $15 \mathrm{~mm}$ aperture diameter) is used as the ultrasound exposure source for all measurements. The Doppler phantom is exposed to the transducer. $16 \mathrm{MHz}$ system clock source is used as the external clock of an arbitrary waveform generator (Lecroy ArbStudio 1104, USA) which generates $2 \mathrm{MHz}$ normal pulse and Barker-coded signal at a pulse repetition frequency (PRF) of $7.8125 \mathrm{kHz}$, with varied pulse lengths (that is, 4, 8, 20 wavelengths). These signals were used to drive transmission circuits via an impedance matching network. Then the transducer transmits different ultrasound waves and receives RF signals to complete all measurements. $8 \mathrm{MHz}$ clock also created by the generator, RF signals and transmitted gate were input to the A/D card (ADLINK PCI-9846) and the Radiofrequency (RF) data were stored in an on-board buffer. The $8 \mathrm{MHz}$ external clock rate determines the sampling frequency $f_{s}$, used by the $\mathrm{A} / \mathrm{D}$ card when digitizing the RF signal after being amplified by pre-amplifier. 


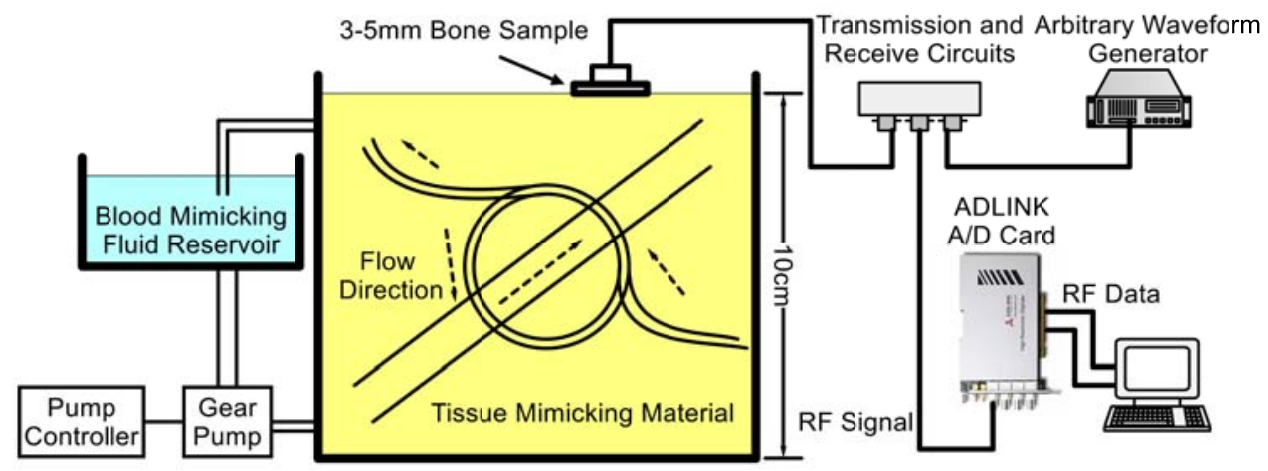

Fig.1 Diagram of experimental system

Coding Principles. In our study, the length of 8 wavelengths base pulse and 7-chip, 11-chip and 13-chip Barker code demodulated by 4 wavelengths base pulse are used as normal pulse and coded excitation respectively. As an example, coding scheme of 7-chip Barker-code demodulated by 4 wavelengths base pulse is illustrated in Figure 2. Where the external clock is the system clock source of $16 \mathrm{MHz}$ and the carrier frequency is $2 \mathrm{MHz}$. These sequences are used to drive the $2 \mathrm{MHz}$ transducer to generate different types of ultrasound waves, after being amplified by the transmission circuits on board. RF signal cable is connected to an AD converter (ADLINK A/D Card) and RF signal is digitized by the A/D card. Then sampled RF data are stored in hard drive and named in a variable in MATLAB environment (MathWorks, MA, USA).

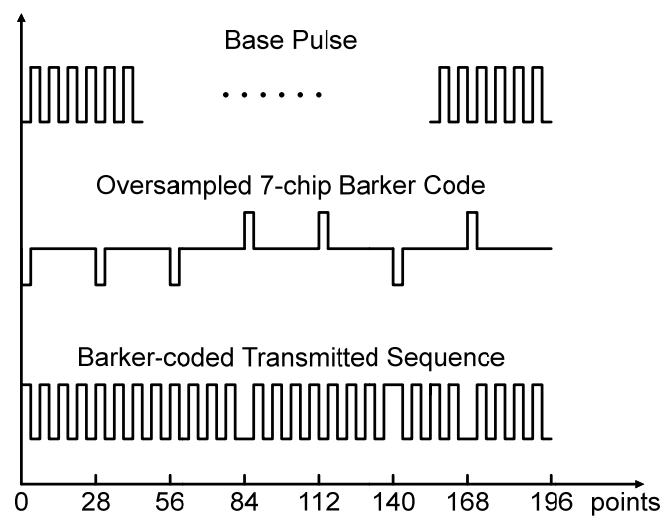

Fig. 2 Processes of coding

Digital I/Q Demodulation. In full digital Doppler ultrasound system, raw RF data instead of the conventional subject of processing, the demodulated Doppler signal, are collected and saved as row vector in a variable named $\mathrm{X}$ after being digitized by AD converter. Then RF data were rearranged into a matrix named $\mathrm{X}_{\mathrm{M} * \mathrm{~N}}$ with the received signal from each transmit burst forming one column.

$$
X_{M \times N}=\operatorname{reshape}(x, M, N)
$$

Where $N$ is the number of the transmit burst and $M$ is the samples every transmit burst. The matrix of $X_{\mathrm{M}^{*} \mathrm{~N}}$ is the subject of processing for the rest of this paper.

In Doppler ultrasound systems, I/Q demodulation is a common and useful RF signal process technique since it allows for the direct extraction of amplitude and phase information. When implemented analog I/Q demodulator in conventional Doppler ultrasound system, plenty of strict demands and inherent errors such as gain balance, DC offsets and impedance match, degrade the performance of it [9]. As $\mathrm{X}_{\mathrm{M}^{*} \mathrm{~N}}$ is digital RF signal, digital software-based I/Q demodulator can be used in this design. Due to the advantages of accuracy, predictability and stability of digital signal processing (DSP) techniques, digital I/Q demodulator using DSP techniques is a successful solution to the disadvantages noted above in conventional analog method. The diagrams of analog conventional and digital I/Q demodulator are compared in figure 3 and figure 4 as well. 


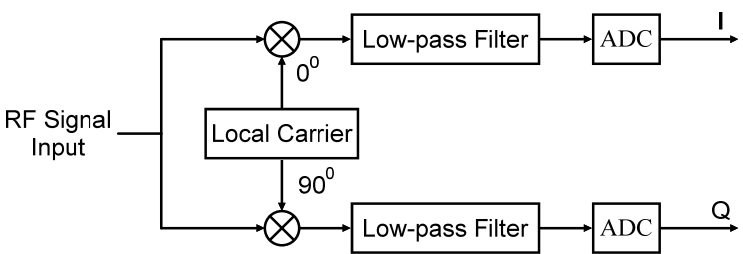

(a)

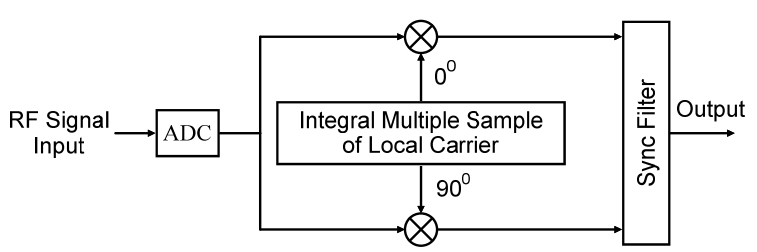

(b)

Fig.3 Conventional I/Q demodulator and Digital I/Q demodulator (a). Conventional I/Q demodulator (b). Digital I/Q demodulator

The process of digital I/Q demodulation can be simplified as an operation of Demod[.]. The data, meanwhile, are down-sampled to the matrix of $\mathrm{Y}_{(\mathrm{M} / 4)} * \mathrm{~N}$, selecting every 4 th element in column. This process is illustrated as equality 2 .

$$
Y_{(M / 4) \times N}=\operatorname{De} \bmod \left[X_{M \times N}\right]
$$

Digital Signal Processing. In non-coded excitation system, $\mathrm{Y}_{(\mathrm{M} / 4){ }^{*} \mathrm{~N}}$ which will be renamed as $Z_{\text {non-coded }}$ can be used for spectrum analysis. If coded excitation, $\mathrm{Y}_{(\mathrm{M} / 4){ }^{*} \mathrm{~N}}$ should be decoded. The decoder, that is the process of cross-correlation indeed, is shown as equality 4.

$$
Z_{\text {coded }}(i, k)=\sum_{j=1}^{M / 4} Y_{(M / 4) \times N}(:, k) c(i+j) \text {. }
$$

Where $i \in[1: M / 4], k \in[1: N] . c(i+j)$ is the oversampled Barker code after zero padding and shifting. $i$ in $c(i+j)$ is the time-shifting parameter of oversampled Barker code sequence. After decoding, $\mathrm{Y}_{(\mathrm{M} / 4)^{*} \mathrm{~N}}$ dimensional matrix of $Z_{\text {coded }}$ is obtained and it will be used for spectrum analysis.

$$
\left\{\begin{array}{l}
S_{\text {non-coded }}=F F T\left[Z_{\text {non-coded }} \cdot W_{T}\right] \\
S_{\text {coded }}=F F T\left[Z_{\text {coded }} \cdot W_{T}\right]
\end{array}\right.
$$

Where FFT[.] is short-time Fourier transform (STFT) operation. $\mathrm{W}_{\mathrm{T}}$ is a window in time domain added to $Z_{\text {non-coded }}$ and $Z_{\text {coded }}$ to reduce the Gibbs effect.

According to the distribution of vessels in the Doppler phantom, the transducer is placed where two or more vessels exist in ultrasound waves' propagation direction. RF signals are collected in vitro from the Doppler phantom using normal pulse and 7-chip Barker-code excitation. $\mathrm{S}_{\text {non-coded }}$ and $\mathrm{S}_{\text {coded }}$ are obtained by processing the RF data with the methods noted above. $\mathrm{S}_{\text {non-coded }}$ and $\mathrm{S}_{\text {coded }}$ which can describe the full-depth blood flow information can be obtained by adding a window of $W_{T}$ in frequency domain to the accumulation of each transmit burst:

$$
\left\{\begin{array}{l}
S_{\text {non-coded }}^{\prime}=S_{\text {non-coded }} \times W_{F} \\
S_{\text {coded }}^{\prime}=S_{\text {coded }} \times W_{F}
\end{array}\right.
$$

\section{Results}

The distribution of full-depth blood flow using normal pulse and 7-chip Barker-code excitation is illustrated in Figure 4. X-axis is depth and Y-axis is relative amplitude of blood flow information. As shown in the figure, two peaks (p1 and p2 labeled in Fig.4 (a) and Fig.4 (b)) mean the blood flow signals of two vessels in different depths of $5 \mathrm{~cm}$ and $7 \mathrm{~cm}$ in ultrasound waves' propagation direction. The results validate that it is feasible to utilize the full digital design in conventional excitation Doppler ultrasound system as well as coded excitation system. Furthermore, full-depth blood flow measurement graph makes it much more convenient for a doctor to focus on a vessel that who is interested in [10]. 


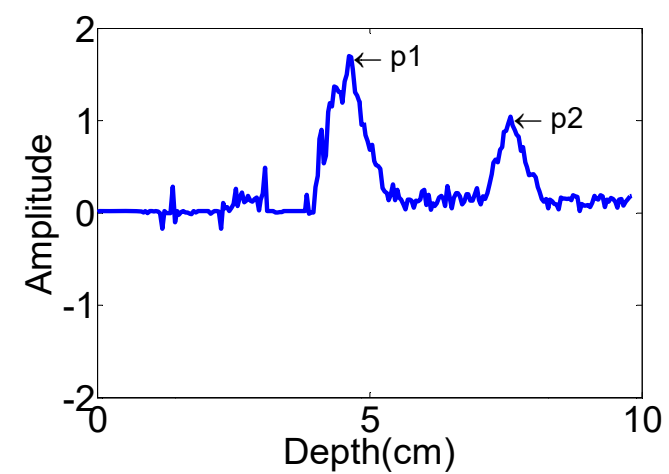

(a)

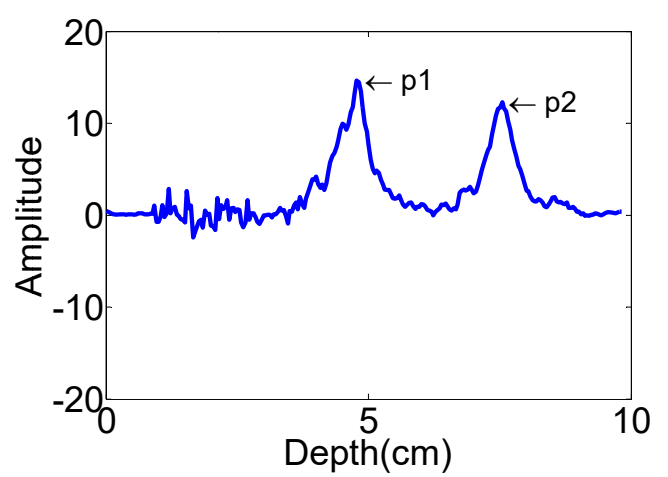

(b)

Fig.4 Full-depth blood flow display. (a). Non-coded excitation. (b). Coded excitation

In order to study the benefits of coded excitation, one vessel is focused on in this experiment. Take the vessel in the depth of some $5 \mathrm{~cm}$ as an example, the advantages of coded excitation can be illustrated in figure 5. With the same normalization factor, the relative amplitude of the blood flow signal using 7-chip Barker-code excitation is over 20 times stronger than that using non-coded excitation. As shown in equality 6 , that is to say, the system gain can be increased by over $20 \mathrm{~dB}$ in average by utilizing coded excitation.

$$
d B=20 \lg \frac{S_{\text {coded }}^{\prime}}{S_{\text {non-coded }}^{\prime}}=27.86
$$

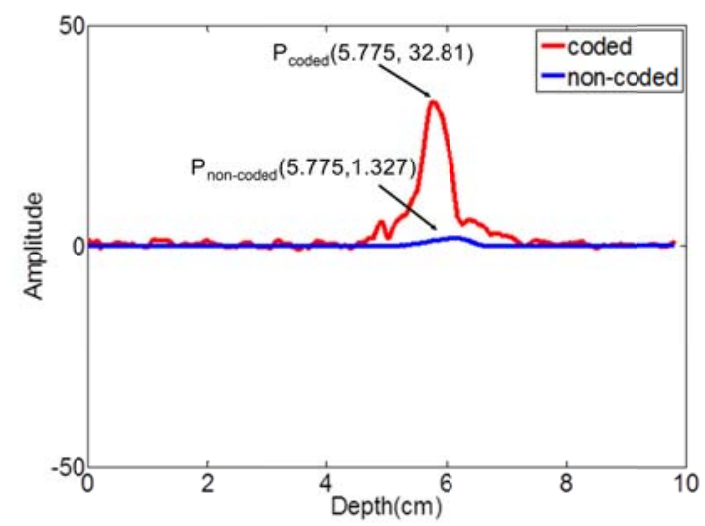

(a)

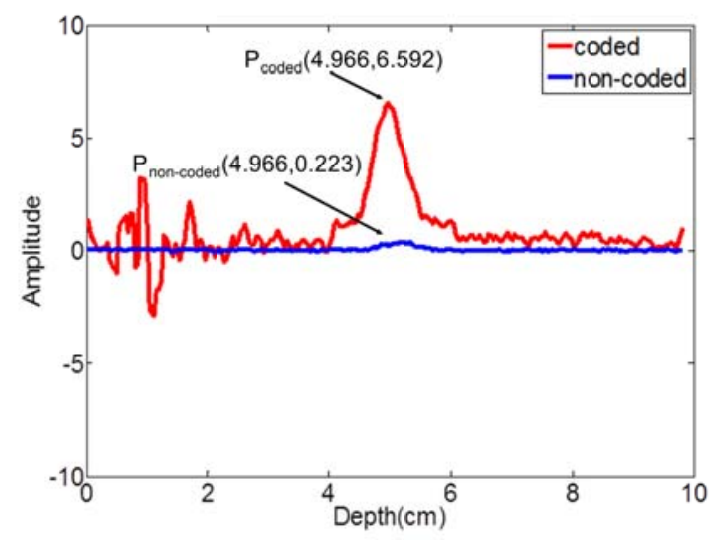

(b)

Fig.5 Blood flow measurement using non-coded and coded excitation (a) No bone is placed between the transducer and Doppler phantom (b) A bone sample is placed between the transducer and Doppler phantom.

In addition, the advantages will be highlighted when the full digital design with coded excitation is used in transcranial Doppler system. The excitation used in transcranial experiments coincides with that used in previous. Figure $5 \mathrm{~b}$ shows the blood flow signal from the same vessel as that in figure 5 after a bone sample is located between the transducer and the Doppler phantom. The blood flow depths displayed in figure $5 \mathrm{a}(5.775 \mathrm{~cm})$ and figure $5 \mathrm{~b}(4.966 \mathrm{~cm})$ are different due to a little difference in locations of the transducer in these two experiments. These results reveal that the system gain can be increased by approximately $30 \mathrm{~dB}$ under the transcranial circumstances utilizing coded excitation. It is also necessary to be noted that the near-field fluctuation in figure 6 is caused by the inferior bonding between the bone sample and the Doppler phantom.

$$
d B_{\text {bone }}=20 \lg \frac{S_{\text {coded }}^{\prime}}{S_{\text {non-coded }}^{\prime}}=29.41
$$




\section{Conclusion}

In summary, a full digital design is proposed to provide full-depth display which can facilitate clinical diagnosis in Doppler ultrasound examination. More than that, under the full digital circumstances, coded excitation can be well used to improve the ultrasound penetration and system SNR. Experiments' results noted above confirm the feasibility of it. The full digital design also offers the rises in system stability, eliminates the system error and reduces the scale of hardware and power consumption because the stable and accurate software-based digital signal processing (DSP) techniques are used. The significance of current work is to explore a new design for Doppler ultrasound system which can provide more adequate information and make measurement much easier for doctors in clinical diagnosis. At present, some clinical experiments have exposed the advantages of the full digital design. In the future, more statistical clinical data will be studied for this design.

\section{Acknowledgements}

This study was supported by National Nature Science Foundation of China (Grants 81371637, 61201041, 61031003), Natural Basic Research Priorities Program of Shenzhen (Grants JC201005280685A), Shenzhen Overseas Innovation Fund Project (Grants KQC201105310020A), Improving Project of Shenzhen Key Laboratory (Grants CXB20110421003A).

\section{Author information:}

The first author, Shaoxing Li, is a graduate of Shenzhen University; And the first corresponding author, Zhengdi Qin, is a professor of Shenzhen University; The second corresponding author, Xin Chen, is an associate professor of Shenzhen University.

\section{References}

[1] M. O'Donnell, "Coded Excitation System for Improving the Penetration of Real-time Phased-array Imaging Systems," IEEE Transactions on Ultrasonics, Ferroelectics, and Frequency Control (UFFC), pp. 341-351, 1992.

[2] Chiao, R.Y. and X.H. Hao, "Coded Excitation for Diagnostic Ultrasound: A System Developer's Perspective," IEEE Transactions on Ultrasonics Ferroelectrics and Frequency Control (UFFC), pp. 160-170, 2005.

[3] Joanne Cowe, John Gittins, A. Ross Naylor, and David H. Evans, "RF Signals Provide Additional Information on Embolic Events Recorded during TCD Monitoring," Ultrasound in Med. \& Biol., Vol. 31, No. 5, pp. 613-623, 2005.

[4] Joanne Cowe, John Gittins, and David H. Evans, "Coded Excitation in TCD Ultrasound Systems to Improve Axial Resolution," Ultrasound in Med. \& Biol., Vol. 33, No. 8, pp. 1296-1308, 2007.

[5] Xiang Lei, Zhao Heng, and Gao Shangkai, "Barker Code in TCD Ultrasound Systems to Improve the Sensitivity of Emboli Detection," Ultrasound in Med. \& Biol., Vol. 35, No. 1, pp. 94-101, 2009.

[6] Qin ZD., Chen SP., Chen X., "Coded Transmission for Ultrasound Doppler Detection Using Truncated Long Code," Biomedical Engineering and Informatics (BMEI), Vol.1, pp.159-161, 16-18 Oct, 2010.

[7] Qin ZD., LIU DQ., Chen SP., "Experimental Study on The Truncated Long Code Sequence for Ultrasound Doppler Detection," 5th International Conference on Biomedical Engineering and Informatics (BMEI), 2012.

[8] Jie LI, etc., "Code Excitation System for Stationary Target Detection Using Multi Segment Coding," 2014 International Conference on Industrial and Information Technology (IEIT), 
pp.395-399, 16-18 May, 2014.

[9] C. Ziomek and P. Corredoura, "Digital I/Q Demodulator," PAC 95, 1996.

[10] Jie LI, Xianfen DIAO, Kai ZHAN, and Zhengdi QIN, "A Full Digital Design of TCD Ultrasound System Using Normal Pulse and Coded Excitation," 1st Global Conference on Biomedical Engineering, 9th Asian Pacific Conference on Medical and Biological Engineering (GCBME/APCMBE), 9-12 Oct, 2014. 